\title{
Local Potential Gradient Developed During Excess Point-Discharge Current
}

\author{
A.K. KAMRA and N.C. VARSHNEYA \\ Physics Department, University of Roorkee, Roorkee, India
}

(Received March 18 ; revised June 17, 1968)

\begin{abstract}
The phenomenon of excess point discharge which reports anomalous values of point discharge for the after-discharge potentials during rapid field-changes, is explained by the development of a transient local potential around the point during such changes. Magnitude of this local potential has been calculated from the concentration of space charge in the field-region of the point. The local potential is found to be $-\frac{V-V_{0}}{4}$ where $V$ is the predischarge potential and $V_{0}$, the critical potential to start point discharge. The theoretical and observed values of point-discharge currents at after-discharge potentials have also been compared.
\end{abstract}

\section{Introduction}

Whenever the electric field in the atmosphere increases to some minimum critical value, point discharge starts at sharp points raised above the earth's surface. It is, naturally, believed that for any value of potential gradient less than the critical, there occurs no pointdischarge current and the sign of the current is the same as that of the potential gradient. Our results of observations of point-discharge current from an artificial sharp point erected in the atmosphere are exactly in conformity of these facts. However, during the rapid fieldchanges, caused by lightning discharges, the nature of variation of point-discharge current with potential gradient and wind speed differs from those of during normal field-changes. As reported in our earlier publication (Kamra and Varshneya, 1967), if the field suddenly decreases very rapidly from an above-critical to a below-critical value, there occurs a pointdischarge pulse. Unlike in the normal field-changes where there would have been no pointdischarge current at the below-critical value of 'after-discharge' potential i.e. the potential difference between the point and the atmosphere just after the occurance of the lightning flash, there is a definite current value at the after-discharge potential during rapid fieldchanges. If the after-discharge potential is above the critical value and opposite in sign to that of 'pre-discharge' potential i.e. the difference of potential between the point and the atmosphere just prior to the occurance of the lightning discharge, then the value of the current at the after-discharge potential is larger than it would have been had this potential been attained slowly, thus the name of 'excess point-discharge current'.

To explain the phenomenon of excess point discharge it is argued that during such rapid field-changes, ions of the sign of the potential gradient are squeezed-out of the fieldregion around the point and the remaining ions of the opposite sign produce a space charge 
and thus a local potential around the point which is opposite in sign to the pre-discharge potential. This local potential is transient in nature and vanishes after giving a current pulse.

Earlier, we assumed the magnitude of this local potential developed around the point, to be equal to the minimum critical value to start point discharge. In this paper we have theoretically calculated the magnitude of this local potential and find that the potential, instead of having a constant value in all cases, depends upon the pre-discharge potential. The assumption taken in the earlier paper, however, comes out to be as a special case of the generalised theory presented here.

\section{Calculation and Discussion}

The local potential, $V_{L}$, should depend on the space charge density $\rho$, round the point at the time of the discharge. Taking the case of positive potential gradient; suppose the pre-discharge potential is $V$ and the critical potential is $V_{0}$. Let $b$ be the distance at which the forces on the ions due to electric field and wind are equal so that a sphere of radius $b$ around the point, separates the field-region from the wind-region. It is assumed that all the space charge is confined within the field-region and is uniformaly distributed.

If $X_{b}$ is the electric field at distance $b$ from the point then the current through the sphere is (in MKS units)

or

$$
\begin{aligned}
& I=4 \pi b^{2} \rho \omega X_{b} \\
& \rho=\frac{I}{4 \pi b^{2} \omega X_{b}}
\end{aligned}
$$

where $\omega$ is the mobility of the ions.

Now if $W$ is the wind velocity

$$
X_{b}=\frac{W}{\omega}
$$

and from Chalmers (1962) relation,

$$
I=-2 \pi \epsilon\left(V-V_{0}\right) W
$$

where $\epsilon$ is the permittivity of free space.

Putting the values of $X_{b}$ and $I$ into (1)

$$
\rho=-\frac{\epsilon\left(V-V_{0}\right)}{2 b^{2}}
$$

Now considering after-discharge state of field-region, if $r$ if any distance from the point such that $r<b$, then the local potential is

$$
V_{L}=\frac{1}{4 \pi \epsilon} \int_{0}^{b} \frac{4 \pi r^{2}}{r} d r \cdot \rho=\frac{1}{2 \epsilon} b^{2} \rho
$$

Putting the value of $\rho$ from (4)

$$
V_{L}=-\frac{V-V_{0}}{4}
$$


It is interesting to note that since $V>V_{0}$, the direction of $V_{L}$ shall always be opposite to that of $V$.

If $V^{\prime}$ is the after-discharge potential then the total potential difference between the point and the atmosphere is

$$
V_{T}=V^{\prime}+V_{L}=V^{\prime}-\frac{V-V_{0}}{4}
$$

Now assuming, as in the earlier paper (Kamra and Varshneya, 1967), that the relation (3) of Chalmers can be applied in the after-discharge conditions as well, the after-discharge current shall be

$$
I^{\prime}=-2 \pi \epsilon W\left(V^{\prime}-\frac{V-V_{0}}{4}\right)
$$

Taking $V$ to be positive, $V^{\prime}$ may be either positive or negative. If $V^{\prime}$ is negative $I^{\prime}$ shall always have direction opposite to that of $I$, but if $V^{\prime}$ is positive then the direction of $I^{\prime}$ shall be determined by whether the local potential is greater or smaller than the after-discharge potential. Thus, if after-discharge current is to have the direction opposite to that of predischarge current, the pre-discharge potential should be sufficient enough to produce local potential greater than the after-discharge potential.

If $V=5 V_{0}$, we get

$$
I^{\prime}=-2 \pi \epsilon W\left(V-V_{0}\right)
$$

The equation is found to be the same as we assumed in our earlier paper, and thus justifies it.

The condition for the pre-discharge and after-discharge currents to be equal, is now modified as below:

For

$$
I=-I^{\prime}
$$

or

$$
\begin{aligned}
& V_{0}-V=V^{\prime}-\frac{V-V_{0}}{4} \\
& V^{\prime}=3 / 4\left(V_{0}-V\right)
\end{aligned}
$$

It shall be wrong to interpret from (8) that the phenomenon of excess point discharge can take place even if after-discharge potential remains above the critical value (without changing direction), because in that case the after-discharge potential itself shall be sufficient to produce fresh ions and thus there shall be no local potential gradient.

\section{Comparison with Observed Values}

Thus we have seen that the squeezing-out of the ions of one sign from the field-region of the point during rapid field-changes gives rise to a local potential around the point which depends upon the pre-discharge potential and is not constant, equal to $V_{0}$, as assumed earlier. If pre-discharge potential is five times the critical potential, $V_{0}$, then the local potential is equal to the critical potential.

Thus the value of after-discharge current shall depend upon the local potential and the after-discharge potential. The after-discharge current may be either positive or negative if 
Table 1

\begin{tabular}{c|c|c|c|c|c|c|c}
\hline \multirow{2}{*}{ Sl. No. } & $V$ & $V^{\prime}$ & $\frac{V-V_{0}}{4}$ & $V^{\prime}-\frac{V-V_{0}}{4}$ & $W$ & $I_{\text {theo }}^{\prime}$ & $I_{\text {obs }}^{\prime}$ \\
& $K V$ & $K V$ & $K V$ & $K V$ & $m / s$ & $\mu A$ & $\mu A$ \\
\hline 1 & +10.62 & -1.06 & +1.66 & -2.72 & 2.8 & -0.43 & -0.6 \\
2 & +8.23 & -2.20 & +1.06 & -3.26 & 2.8 & -0.51 & -1.0 \\
3 & +12.47 & -0.80 & +2.12 & -2.92 & 2.8 & -0.46 & -0.47 \\
4 & +12.47 & 0 & +2.12 & -2.12 & 2.8 & -0.33 & -0.43 \\
5 & -10.62 & 0 & -1.66 & -1.66 & 1.8 & +0.16 & +0.15 \\
6 & -9.82 & +6.4 & -1.46 & -7.86 & 0.9 & +0.39 & +0.33 \\
7 & +12.74 & -3.45 & +2.19 & -5.64 & 0.9 & -0.29 & -0.5 \\
8 & +12.74 & -4.7 & -2.19 & -6.89 & 1.8 & -0.7 & -1.0 \\
9 & -5.57 & +3.98 & -0.07 & -4.05 & 5.5 & +1.25 & +2.0 \\
10 & -8.50 & +9.3 & -0.80 & -10.1 & 5.3 & +3.00 & +3.00 \\
11 & -14.07 & -2.1 & -2.19 & -0.09 & 0.9 & +0.45 & +0.7 \\
12 & -14.87 & +5.31 & -2.39 & -7.70 & 4.5 & +1.94 & +3.00 \\
13 & -13.81 & -0.80 & -2.13 & -1.33 & 2.8 & +0.21 & +0.45 \\
14 & -12.48 & -0.26 & -1.79 & -1.53 & 2.8 & +0.24 & +0.9 \\
\hline
\end{tabular}

pre-and after-discharge potentials are of the same polarity depending upon whether local potential is larger or lesser than the after-discharge potential. If, however, the afterdischarge potential differs in sign from the pre-discharge potential, the after-discharge current shall always be of opposite sign to that of the pre-discharge current. This result is a modification over that given in our earlier paper where the after-discharge current was reported to be always of opposite polarity to that of pre-discharge current.

Table 1 gives the observed and calculated (from (8)) values of point-discharge current at after-discharge potentials. The values of $V_{0}$ are $3.98 \mathrm{KV}$ and $5.31 \mathrm{KV}$ for positive and negative currents respectively. It is clear that while in some cases the two values differ significantly, in general there is fair agreement considering that the values correspond to individual cases and are not statistically rich. Many more cases were available but in them either the pre-discharge or after-discharge values of potential gradient or point-discharge current have gone out of scale used at that time. However, larger statistics of such events can better confirm the coherence of theoretical and observed values.

\section{Acknowledgements}

We wish to thank Uttar Pradesh Government and Council of Scientific and Industrial Research, New Delhi, for financial grants to carry out this work.

\section{References}

Chalmers J.A., The relation of point discharge current to potential difference and wind speed., $J$. Atmosph. Terr. Phys., 24, 339-344, 1962.

Kamra A.K. and Varshneya N.C., Excess point-discharge currents during rapid field-changes., $J$. Atmosph. Terr. Phys., 29, 1519-1527, 1967. 